\title{
Patient Flow, Health Delivery Processes, and Areas for Improvement in the UP Health Service (UPHS) during May to June 2020 of the COVID-19 Pandemic
}

\author{
Shiela Marie S. Laviña, MD, Marishiel Mejia-Samonte, MD, Geannagail O. Anuran, MD, \\ Katrina Lenora Villarante, MD and Anna Guia O. Limpoco, MD \\ Department of Family and Community Medicine, Philippine General Hospital, University of the Philippines Manila
}

\begin{abstract}
Background. Workplace or employees' clinics play a vital role in disease outbreaks as there could be an influx of sick personnel. Processes and patient flows during pandemics should be documented to identify good practices and sources of operational inefficiencies.

Objective. To describe the patient flow, health delivery processes, and areas for improvement at the UPHS during the early phase of the COVID-19 pandemic from May to June 2020.

Methods. This was a cross-sectional study involving patient flow analysis of processes at the employees' clinic of the University of the Philippines-Philippine General Hospital. The study was divided into two major components: clinic process time measurement and process flow mapping. Data collection involved time elements and narrative descriptions of good practices and problems in the process flow.

Results. The UPHS staff attended to 1,514 employees' visits during the 15 working days from May to June 2020. The total UPHS service time from arrival to end of consultation of an employee with a COVID-19-related concern was an average of 1 hour 3 minutes (SD \pm 39 minutes) with a mean total waiting time of 46 minutes (SD \pm 37 minutes). Good practices identified were personnel flexibility in doing other tasks, good communication, and infection control measures. Areas for improvement included symptom screening, implementation of physical distancing, and disinfection practices.

Conclusion. The process flows in the UPHS clinic consisted of COVID-19 related consultations, non-COVID-19 related concerns, and swabbing services. Good communication, staff flexibility, infection control measures, and leadership were identified as good practices. Occasional lapses in symptom screening at triage, physical distancing among employees in queuing lines, and inconsistent disinfection practices were the areas for improvement.
\end{abstract}

Key Words: patient flows, health care delivery, COVID-19

\section{INTRODUCTION}

Workplace or employees' clinics play a vital role during pandemics when there is an increase in sick personnel. The sudden influx of sick health workers increases the demand for services and generates problems in clinic processes and patient flows. However, health care facilities sometimes lack the necessary protocols for health service delivery during outbreaks.

The UP Health Service (UPHS) provides medical Corresponding author: Shiela Marie S. Laviña, MD Department of Family and Community Medicine Philippine General Hospital University of the Philippines Manila

Taft Avenue, Ermita, Manila 1000, Philippines Email:sslavina@up.edu.ph services to the Philippine General Hospital's healthcare workers. The clinic had an increased number of consultations during the COVID-19 pandemic. Changes were made in the physical setup, staffing, resources, and patient care 
processes to ensure that the health care workers' emerging needs were met while maintaining the baseline clinic services. The infectious nature of COVID-19 warranted that UPHS created parallel patient flows to segregate employees with suspected infections from those who were uninfected but needed other medical services.

During pandemics, health care delivery processes should be evaluated to identify good practices and find sources of operational inefficiencies. The documentation of the current UPHS system through patient flow analysis could improve employees' health service delivery, consequently affecting patient care.

The study's objective was to describe the patient flow, health delivery processes, good practices, and areas for improvement at the UPHS during the May to June 2020 COVID-19 pandemic.

\section{METHOD}

\section{Study Design, Population, and Sample Size}

This was a cross-sectional study on patient flow analysis at the employees' clinic of the University of the PhilippinesPhilippine General Hospital. The study was divided into two major components: time data on clinic visits and narrative descriptions of the processes. All UPHS visits for consult and swabbing were included in the study, while preliminary clinic consultations were excluded.

All consultations of health care workers (HCW) during the study period were included; hence no sample size computation and sampling were done.

\section{Data Collection}

Quantitative and qualitative data were gathered. Research assistants collected the time elements of the study. Simultaneously, the narrative on the process flow was documented as field notes by non-duty senior residents of the Department of Family and Community Medicine (DFCM).

A structured guide was used to observe predetermined patient flow processes for non-COVID-19 consultation, COVID-19 consultation, and swabbing. Quantitative data, including arrival, start, and end times in each step, was documented using a patient flow time log. The research assistants were trained on the data collection process. The UPHS process flow during the May/ June 2020 COVID-19 pandemic is diagrammatically represented in Figure 1.

\section{Study Outcomes and Data Analysis}

The quantitative study outcomes included time intervals on service delivery processes at the non-COVID-19, COVID-19, and swabbing areas. Waiting and service times were documented in minutes at the three clinic areas. The qualitative outcomes were a narrative description of clinical processes, administrative activities, staffing requirements, good practices, operational inefficiencies, and variations from the standard protocols.

All quantitative data were analyzed using the data analysis function of Microsoft Excel Version 16.16.21. Time intervals were computed using descriptive statistics for each step of the UPHS visit. Qualitative data based on field notes were analyzed using content analysis. Open coding was used for all observations using MAXQDA. The ideas based on the field notes were aggregated to form common themes and then summarized into categories.

\section{Ethical Considerations}

The study was exempted from the Ethics Review Board of the University of the Philippines-Philippine General Hospital (UPREB) with code number 2020-305-EX.

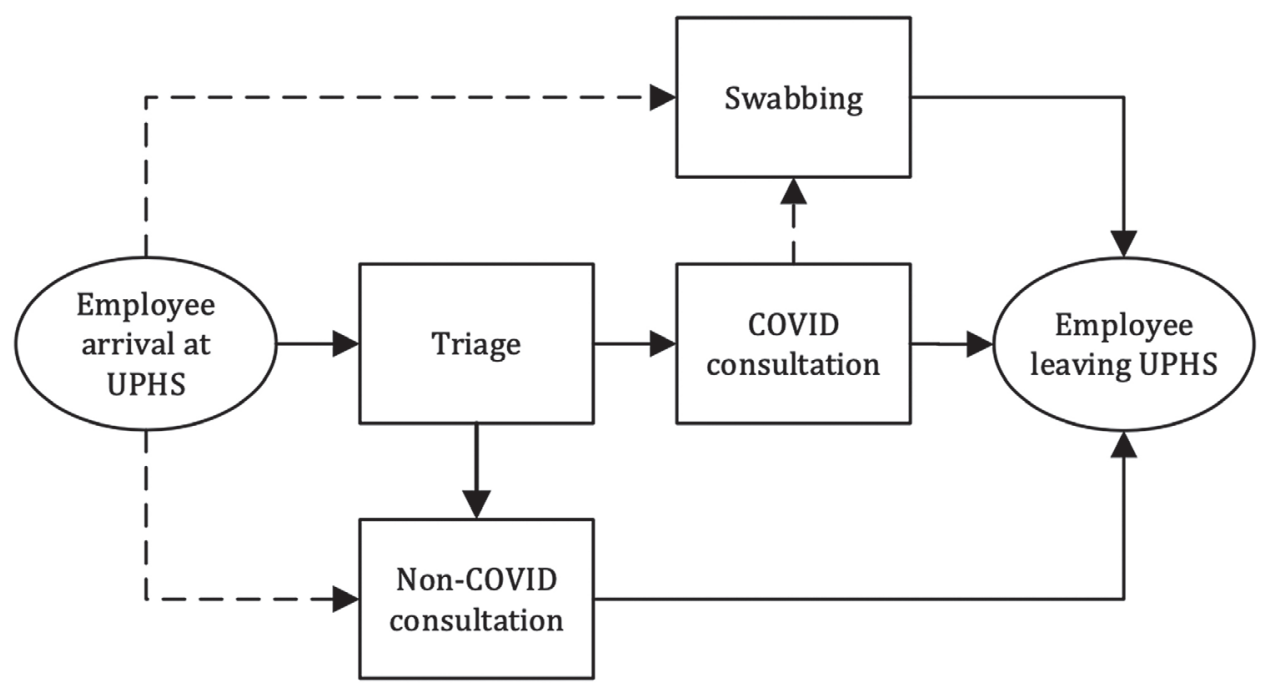

Figure 1. Process Flow of Employees' Consult at UPHS. 


\section{RESULTS}

The UPHS staff attended 1,509 employees' visits during the 15 working days of May to June 2020 COVID-19 pandemic. There were three process flows in the UPHS clinic: COVID-19 consultations, non-COVID-19 concerns, and swabbing services (Figure 1).

\section{Process Flow in the UPHS}

The clinic visits process flow in the non-COVID-19 area included screening, pre-consultation assessment, consultation, and post-consult advice. There were 718 nonCOVID-19-related clinic visits, with an average of 47 employees seen per day. The non-COVID-19 service area catered to 445 employees who needed face-to-face medical consultation and 268 who claimed fit-to-work certification issued through telemedicine. On the other hand, the COVID-19 area, consisting of the triage and consultation, served 351 employees.

The swabbing process began with verifying the submitted Case Investigation Form (CIF), followed by specimen collection and information on the release of results. A total of 377 tests were done, with an average of 25 employees swabbed per day.

For non-COVID-19 consultations, the human resources complement included one nursing assistant in the screening area, 2-3 nurses conducting the pre- and post-consultations, and two physicians for the consultation proper. In the COVID-19 consultation area, one nurse was assigned to triage while four physicians conducted the consultations. For the swabbing area, one clinic staff (often a doctor, sometimes a nurse) was stationed in the receiving booth, while two swabbing booths were each operated by 1-2 physicians (including residents from other specialties such as ORL), with 1-2 nurses assisting in the swabbing booths.

\section{Waiting and Service Time}

The COVID-19 triage waiting time was at an average of 16 minutes ( $\mathrm{SD} \pm 21$ minutes), while the mean triage time was 3 minutes ( $\mathrm{SD} \pm 4$ minutes). The median waiting time for consultation was 37 minutes, while the average consultation time was 14 minutes ( $\mathrm{SD} \pm 9$ minutes) per employee. The average total service time for COVID-19-related concerns from arrival to the consultation was 1 hour 3 minutes (SD \pm
39 minutes), with a mean total waiting time of 43 minutes ( $\mathrm{SD} \pm 37$ minutes) (Table 1$)$.

In the non-COVID-19 area, the average waiting time for the issuance of a pre-printed medical certificate was 1 minute (SD \pm 3 minutes), while the mean waiting time for consultation was 7 minutes ( $\mathrm{SD} \pm 11$ minutes). Consultation time was at an average of 6 minutes ( $\mathrm{SD} \pm 5$ minutes), while post-consultation advice by a nurse was given at a mean time of 2 minutes ( $\mathrm{SD} \pm 8$ minutes) (Table 2). Overall, the UPHS average service time for non-COVID-19 consults was 37 minutes ( $\mathrm{SD} \pm 25$ minutes) and a total waiting time of 12 minutes ( $\mathrm{SD} \pm 19$ minutes) (Table 1 ).

Waiting time for swabbing was at an average of 33 minutes ( $\mathrm{SD} \pm 32$ minutes), with a mean swabbing time of 2 minutes ( $\mathrm{SD} \pm 1$ minute) (Table 2). The total UPHS service time for swabbing was 35 minutes ( $\mathrm{SD} \pm 32$ minutes), with a median of 24 minutes (Table 1 ).

\section{Good Practices Observed}

The UPHS physical setup, service delivery process flows, and staffing underwent rapid changes as infection control and prevention became a priority due to the pandemic. Onsite patient services of UPHS were provided in three separate areas, namely COVID-19, non-COVID-19 consultation, and swabbing. The COVID-19 consultation and swabbing areas were located in open, well-ventilated spaces. Transparent plastic barriers were placed in booths. Seats were spaced one meter apart in the waiting rooms to ensure physical distancing between patients. Areas were designated for donning and doffing of personal protective equipment (PPE). Alcohol dispensers were placed in the COVID-19 consultation and swabbing stations for both the staff and patients. Disinfection of phones, barriers, and other surfaces was done daily before clinic opening. Consultation booths and swabbing stations were also sanitized between patient encounters and after clinic hours. The wearing of appropriate levels of PPE required in specific areas of the clinic was also observed.

Service delivery process flows were also modified in the UPHS. The screening booth at the non-COVID-19 area addressed inquiries about clinic services, stamping of laboratory request/sick leave forms, and releasing medical certificates for monitored employees, then cleared via telemedicine. Assessment of required documents for pre-

Table 1. Total Service and Waiting Time in the Non-COVID, COVID and Swabbing Areas of the University of the Philippines Health Service (UPHS) during the 15-day Study Period in May to June 2020 COVID-19 Pandemic

\begin{tabular}{lcccc} 
& Covid Clinic & \multicolumn{2}{c}{ Non-Covid Clinic } & Swabbing Booth \\
$\mathbf{N}=\mathbf{3 5 1}$ & & $\begin{array}{c}\text { Issuance of Certificates } \\
\mathbf{N}=\mathbf{2 6 8 * *}\end{array}$ & $\begin{array}{c}\text { Consultations } \\
\mathbf{N}=\mathbf{4 4 5 *}\end{array}$ & \begin{tabular}{c} 
Parameters \\
\cline { 3 - 5 }
\end{tabular} \\
Waiting time, in minutes & $45.72( \pm 36.62)$ & $1.12( \pm 3.05)$ & $11.77( \pm 19.12)$ & $33.38( \pm 31.81)$ \\
Service time, in minutes & $62.96( \pm 39.2)$ & $4.64( \pm 7.07)$ & $25.32( \pm 18.40)$ & $2.09( \pm 1.18)$ \\
Total Length of stay at service area & $\mathbf{1 0 8 . 6 8 ( \pm 7 4 . 9 3 )}$ & $\mathbf{5 . 7 7 ( \pm 7 . 7 9 )}$ & $\mathbf{3 7 . 0 9 ( \pm 2 5 . 2 9 )}$ & $\mathbf{3 5 . 4 7}( \pm 31.84)$ \\
\hline
\end{tabular}

${ }^{*}$ there will be differences in total $N$ due to cases that are not applicable and with missing data ${ }^{* *}$ issuance of certificate $=$ with time from arrival to queuing for release of documents. 
Table 2. Service and Waiting Time in the Non-COVID, COVID and Swabbing Areas of the University of the Philippines Health Service (UPHS) during the 15-day Study Period in May to June 2020 COVID-19 Pandemic

\begin{tabular}{|c|c|c|c|c|}
\hline Parameters & Overall & Week $1(\mathrm{~N}=230)^{*}$ & Week $2(\mathrm{~N}=253)^{*}$ & Week $3(\mathrm{~N}=236)^{*}$ \\
\hline \multicolumn{5}{|l|}{ Covid Clinic, $N=351$} \\
\hline \multicolumn{5}{|l|}{ Triage } \\
\hline Triage waiting time & $16.13( \pm 20.87)$ & $14.21( \pm 19.96)$ & $16.78( \pm 21.20)$ & $17.00( \pm 21.31)$ \\
\hline Triaging time & $2.96( \pm 3.65)$ & $2.14( \pm 4.83)$ & $2.64( \pm 1.47)$ & $4.43( \pm 4.53)$ \\
\hline \multicolumn{5}{|l|}{ Consultation } \\
\hline Waiting time & $42.74( \pm 36.90)$ & $56.39( \pm 48.67)$ & $36.87( \pm 25.27)$ & $38.91( \pm 36.94)$ \\
\hline Consultation time & $14.17( \pm 9.45)$ & $21.05( \pm 12.22)$ & $9.28( \pm 4.40)$ & $16.94( \pm 7.89)$ \\
\hline \multicolumn{5}{|l|}{ Non-Covid Clinic, $N=718$} \\
\hline \multicolumn{5}{|l|}{ Issuance of Certificates $(\mathrm{N}=265)^{*}$} \\
\hline Waiting time: arrival to queuing** & $1.12( \pm 3.05)$ & $1.48( \pm 3.85)$ & $0.90( \pm 2.76)$ & $0.87( \pm 1.84)$ \\
\hline Service time: releasing of documents & $4.64( \pm 7.04)$ & $4.64( \pm 8.77)$ & $3.37( \pm 3.65)$ & $6.08( \pm 7.05)$ \\
\hline \multicolumn{5}{|l|}{ Consultations $(\mathrm{N}=428)^{*}$} \\
\hline Waiting time: arrival to screening & $2.16( \pm 7.98)$ & $1.40( \pm 3.91)$ & $2.94( \pm 9.65)$ & $1.88( \pm 8.25)$ \\
\hline Screening time & $10.07( \pm 16.10)$ & $6.88( \pm 11.69)$ & $10.77( \pm 17.29)$ & $11.78( \pm 17.41)$ \\
\hline Pre-consultation waiting time & $2.31( \pm 12.14)$ & $2.03( \pm 10.83)$ & $2.09( \pm 9.56)$ & $2.78( \pm 15.33)$ \\
\hline Pre-consultation (Nurse/Vital Signs) & $6.66( \pm 3.69)$ & $623( \pm 4.06)$ & $6.47( \pm 3.09)$ & $7.18( \pm 3.96)$ \\
\hline Consultation waiting time & $6.63( \pm 11.24)$ & $3.95( \pm 6.33)$ & $4.66( \pm 9.14)$ & $10.84( \pm 14.58)$ \\
\hline Consultation time (Physician) & $6.49( \pm 4.67)$ & $7.45( \pm 6.42)$ & $6.64( \pm 4.08)$ & $5.57( \pm 3.34)$ \\
\hline Post-Consultation waiting time & $0.45( \pm 2.53)$ & $0.17( \pm 0.40)$ & $0.27( \pm 0.87)$ & $0.87( \pm 4.14)$ \\
\hline Post-Consultation (Nurse/Advise) & $2.08( \pm 8.30)$ & $3.62( \pm 15.70)$ & $1.39( \pm 1.75)$ & $1.66( \pm 2.17)$ \\
\hline \multicolumn{5}{|l|}{ Swabbing Booth, in minutes, $N=377$} \\
\hline Waiting time in queuing line & $33.38( \pm 31.81)$ & $39.16( \pm 31.17)$ & $38.20( \pm 33.15)$ & $14.03( \pm 20.66)$ \\
\hline Swabbing time & $2.09( \pm 1.18)$ & $2.73( \pm 1.30)$ & $2.17( \pm 0.70)$ & $0.91( \pm 0.92)$ \\
\hline
\end{tabular}

${ }^{*}$ there will be differences in total $N$ due to cases that are not applicable and with missing data ${ }^{*}$ issuance of certificate $=$ with time from arrival to queuing for release of documents ${ }^{*}$ Example $N=715$ : Arrival to screening - no data on 3 employees who were not in the master list and did not pass the screening booth

employment and fit-to-work clearance were done before the consultation.

Queuing was based on patient arrival time for both the COVID-19 and non-COVID-19 areas, while staggered appointment times were the basis in the swabbing area. Walkin patients for the Reverse Transcriptase Polymerase Chain Reaction (RT-PCR) test were accommodated depending on the number of remaining kits. A single or parallel queueing system was used depending on the number of booths open for consultation. Online submission of an accomplished Case Investigation Form (CIF) and the use of a calendar to aid patients in recalling dates of symptom onset and last exposure facilitated the COVID-19 consultation process. Patients were guided on new clinic processes, service locations, and safety protocols through verbal instructions from the staff and visual reminders.

Flexible staffing and good communication were essential for the organized delivery of health services. Personnel from other units in the hospital were assigned to UPHS to augment the staffing requirement. The UPHS staff's adaptability in taking various tasks enabled the clinic to meet the changing staffing demand in the different areas. A staggered schedule of staff breaks ensured continuous service delivery. A physician team leader and head nurse assigned per week conducted day-to-day clinic management, including meetings and timely discussions to address issues encountered. The use of two-way radios facilitated communication among staff in specific areas of the clinic.

\section{Areas for improvement}

The daily operations of the UPHS clinic were observed to have some lapses in the implementation of infection control, staff performance, communication, and coordination. Gaps in infection control procedures included inconsistent hand hygiene, poor donning and doffing practices, the wrong level of PPE used, omission of symptom screening, missed disinfection between patient encounters, and inadequate physical distancing. There was no safety officer solely responsible for ensuring compliance of staff to proper donning and doffing technique. Employees were unable to keep an appropriate distance during surges due to insufficient waiting areas. Patients rearranged chairs to avoid direct sun exposure or gathered in groups with other employees. The clinic staff was inconsistent in giving reminders to maintain the recommended physical distance. COVID-19 consultation physicians sometimes communicated with patients outside of the transparent barrier rendering the booth for infection control.

Clinic staff performance that needed improvement were punctuality, familiarity with protocols, and communication with patients. Personnel occasionally came late for duty or were delayed in starting the clinic. The COVID-19 consultation area was sometimes unmanned in the afternoon. Endorsements between the staff were noted to be absent sometimes, and inconsistencies in giving patient advice and instructions have been observed. Newly assigned staff were unfamiliar with protocols and were unable to address 
patient concerns promptly. Some of the signages were not visible to the patients; hence they tend to wait in the wrong areas. Verbal instructions provided by staff regarding UPHS process flow were unclear, resulting in patients presuming the next steps or following the wrong process.

\section{DISCUSSION}

COVID-19-related consultations, non-COVID-19related visits, and swabbing services were the three process flows in the UPHS clinic. The total service time for COVID19-related consults was more than one hour, while there was a thirty-minute service time for non-COVID-19 consultation and swabbing procedure.

The health service delivery processes within the UPHS needed to quickly respond to the infection control measures demanded by the pandemic, along with the unprecedented number of health workers consulting for respiratory symptoms and exposure to a confirmed COVID-19 case. The clinic continued to provide standard medical care and adjusted its services to meet employees' new needs. The fear of infection and abidance to hospital protocols had prompted HCWs to seek early consultation.

Health care workers are usually at high risk during pandemics. In the 2009 H1N1 influenza pandemic in Korea, physicians and nurses were found to have the highest infection rate. ${ }^{1}$ The COVID-19 pandemic affected 3,019 Chinese health workers and accounted for $3.83 \%$ of total infections by February $2020 .^{2}$ Locally, as of November 29, 2020, 12,425 $(2.89 \%)$ of all the confirmed cases $(\mathrm{N}=429,864)$ and 76 (0.91\%) of the total deaths $(\mathrm{N}=8,373)$ from COVID-19 were health workers. ${ }^{3}$

UPHS is an outpatient clinic for more than 10,000 employees, and the COVID-19 pandemic has changed the way health services were delivered. The clinic had transferred locations and rearranged its physical set-up twice in four months to better accommodate surges in consultations and enforce infection control measures. Its service delivery areas were divided to accept both COVID-19-related and non-COVID-19-related consultations. UPHS information and communication infrastructure have also improved with electronic health records, internet access, cellular phones, and computers. Swabbing services have been transferred from UPHS to the Department of Laboratories. The process in the issuance of fit-to-work clearances was likewise modified, resulting in shorter service times. On the other hand, the number of non-COVID-19 consultations was more than COVID-19 related visits during the data collection period despite the pandemic situation. This showed employees' constant need for uninterrupted delivery of other primary care services even with the growing concerns on COVID-19.

The staff's ability to take on other tasks when there were inadequate human resources in other units were identified as good practices. Hence, there was no disruption of health service delivery due to human resource shortages due to absences. This flexibility lessened the operational inefficiencies in the clinic. Likewise, a team leader who addressed problems promptly contributed to the organized operations of the UPHS. The observed lapses in infection prevention measures, such as missed symptom screening and inadequate physical distancing among the employees seeking consultation, were identified as areas for improvement. These omissions can have dire consequences as COVID-19 transmission remains through respiratory droplets, screening, and physical distancing as some of the best prevention and control measures. ${ }^{4}$

This study was a patient flow analysis (PFA) of UPHS services to document the flow of care and the changes or inefficiencies during clinic visits. A PFA shows the system's ability to serve patients quickly and efficiently as they move through stages of care..$^{5-8}$ Adjustment and improvements in health delivery can be implemented based on the findings of a PFA. A hospital ambulatory clinic analysis of patient flow led to health service delivery improvement by implementing fewer patient stops, better communication among staff, regular brief meetings, and pre-assessment of patient schedules. ${ }^{9}$ In the present study, good practices and areas for improvement were identified and could serve as the basis for recommendations for better delivery of services in UPHS.

Pandemics bring changes to health service delivery processes. The UPHS, as a health facility, was able to institute actions to address infection control, protective equipment, staff scheduling, barrier precautions, disinfection recommendations, venues for medical consultations, and monitoring for illnesses. The clinic processes have been modified to adapt to changing hospital policies, protocols, and employees' needs.

The study limitations include the overall generalizability and applicability of the results to employees' clinics of other institutions. Though lessons can be learned from the study, the physical set-up, hospital policies, and protocols vary by institution, making service delivery unique for each employees' clinic. Likewise, the results' applicability may be limited to tertiary health facilities with surge capabilities in infrastructure and human resources.

Health care workers and employees' clinics are at the frontline during pandemics. A sustainable and suitable pandemic response plan for the UPHS should be formulated to address health workers' needs adequately. Hence, the study recommendations include strengthening the clinic's capacity to accommodate increasing employees' consultations, infrastructure, and data needs. Regular training for the UPHS staff to safely handle outbreaks or epidemics and activate a response plan should be prioritized. There should be a continuous upgrade of information and communication technology to cope with the more significant data requirement and fully utilize telemedicine in monitoring and consultations. Scheduling of patient visits using an online appointment platform must strictly be implemented for better process flow and manage the number of patients in the clinic. 


\section{CONCLUSION}

The process flows in the UPHS clinic include COVID19-related consultations, non-COVID-19 related concerns, and swabbing services. Effective communication, the staff's flexibility in performing other tasks, infection control measures, and leadership were identified as good practices. Occasional lapses in symptom screening at triage, physical distancing among employees in queuing lines, and inconsistent disinfection practices were the areas needing improvement.

\section{Statement of Authorship}

All authors participated in the data collection and analysis and approved the final version submitted.

\section{Author Disclosure}

All authors declared no conflicts of interest.

\section{Funding Source}

The study proposal was a recipient of the PGH Urgency Grant for COVID-19 related research.

\section{REFERENCES}

1. Yeom JS, Lee JH, Bae IG, Oh WS, Moon CS, Park KH, et al. 2009 $\mathrm{H} 1 \mathrm{~N} 1$ influenza infection in Korean healthcare personnel. European journal of clinical microbiology \& infectious diseases. 2011 Oct 1;30(10):1201-6. DOI: 10.1007/s10096-011-1213-2
2. Wang Y, Wang Y, Chen Y, Qin Q. Unique epidemiological and clinical features of the emerging 2019 novel coronavirus pneumonia (COVID-19) implicate special control measures. Journal of medical virology.2020 Jun;92(6):568-76 DOI: 10.1002/jmv.25748 Date Searched/Accessed August 2020.

3. Department of Health. Beat COVID-19 Today: A COVID-19 Philippine Situationer (Issue 217). 2020 November 30. [Internet]. [date cited]. Available from https://app.adpc.net/sites/default/files/ public/publications.

4. World Health Organization. Scientific Brief. Transmission of SARSCOV2: Implications for Infection Prevention Precautions. 2020 July 9.

5. Palvannan RK and Teow KL. Queuing for Healthcare. J Med Syst 2012 36:541-547. DOI 10.1007/s10916-010-9499-7

6. Dixon CA, Punguyire D, Mahabee-Gittens M, Ho M, Lindsell CJ. Patient flow analysis in resource-limited settings: a practical tutorial and case study. Global Health: Science and Practice. 2015 Mar 1;3(1):126-34. https://doi.org/10.9745/GHSP-D-14-00121

7. Almeida R, Paterson WG, Craig N, Hookey L. A patient flow analysis: identification of process inefficiencies and workflow metrics at an ambulatory endoscopy unit. Canadian Journal of Gastroenterology and Hepatology. 2016; 2016:2574076 DOI: 10.1155/2016/2574076

8. Potisek NM, Malone RM, Shilliday BB, Ives TJ, Chelminski PR, DeWalt DA, Pignone MP. Use of patient flow analysis to improve patient visit efficiency by decreasing wait time in a primary care-based disease management programs for anticoagulation and chronic pain: a quality improvement study. BMC health services research. 2007 Dec 1;7(1):8. DOI: 10.1186/1472-6963-7-8

9. Dhar S, Michel R, Kanna B. Improving Visit Cycle Time Using Patient Flow Analysis in a High-Volume Inner-City HospitalBased Ambulatory Clinic Serving Minority New Yorkers. Journal for healthcare quality. 2011 Mar;33(2):23-8. DOI: 10.1111/j.19451474.2010.00111.x

\section{The Acta Medica Philippina is now accepting limited advertising for its front and back cover (colored), as well as for available spaces in some of its pages, as appropriate. For inquiries and submission of proposals, please email us at actamedicaphilippina.upm@up.edu.ph}

